\title{
Treating Osteoarthritic Joints Using Dextrose Prolotherapy and Direct Bone Marrow Aspirate Injection Therapy
}

\author{
Ross Hauser* and Barbara Woldin
}

Caring Medical and Rehabilitation Services, S.C. 715 Lake St., Ste. 600, Oak Park, IL 60301, USA

\begin{abstract}
Osteoarthritis is a chronic, progressive disease of the articular joints, and to date, has no cure or effective longterm treatment. Objective: To determine if bone marrow prolotherapy (BMP), a combined treatment protocol employing separate injections of hypertonic dextrose prolotherapy and bone marrow aspirate, would be effective as a means of reducing joint pain and improving function in osteoarthritic joints. Design: Patients with a clinical diagnosis of radiographic osteoarthritis who visited our pain clinic and underwent BMP treatments $(\mathrm{N}=24$, mean age 64.9) were asked to complete a questionnaire assessing their condition before and after treatment. Methods: BMP treatments (average 3.6) were conducted at 6 to 8 week intervals and involved autologous harvesting and aspiration of the patient's tibial bone marrow, after which a hypertonic dextrose solution was injected at sites in and around the index joint (prolotherapy), followed by injections of the bone marrow aspirate directly into and around the joint. At 6 months post-treatment, patients were e-mailed a questionnaire asking them to rate their condition before and after BMP treatment in terms of pain levels at rest, performing activities of daily living, and during exercise (Visual Analog Pain Scale), as well as their degree of stiffness, range of motion, and level of crepitus. Changes in the self-reported scores of these variables for each patient were analyzed to determine the effectiveness of BMP treatment. Data were obtained by comparing the differences between baseline and posttreatment scores and analyzed utilizing a two-tailed paired $\mathrm{t}$ test. Results: Patient-reported improvements in pain relief and joint function were statistically significant $(P<.001)$, as were gains in activities of daily living, exercise ability, and range of motion and losses in stiffness and crepitus. No adverse events occurred. Conclusion: Our survey of patientreported outcomes supports the use of BMP as an effective therapy for treating osteoarthritis and suggests that BMP has potential for enhancing the quality of life of individuals with the disease.
\end{abstract}

Keywords: Bone marrow aspirate, cartilage, dextrose prolotherapy, injection therapy, osteoarthritis.

\section{INTRODUCTION}

Osteoarthritis $(\mathrm{OA})$ is the most common joint disease in the world, affecting approximately 27 million people in the United States alone, and represents the majority of the $\$ 128$ billion cost burden Americans incur each year on arthritisrelated health care [1]. The World Health Organization ranks $\mathrm{OA}$ as the fourth leading cause of 'years lived with disability' (YLDs), accounting for 3\% of total global YLDs, stating that $80 \%$ of those with OA will experience limitations in movement over time, and $25 \%$ will be unable to perform routine daily activities of life $[2,3]$. Overall, an estimated 70 million people over age 65 will be at risk for OA by 2030, according to data released by the Osteoarthritis Initiative (OAI), a multicenter, longitudinal, prospective observational study of knee OA $[4,5]$. Due to the aging of America and the endemic increase in obesity, these figures are expected to grow exponentially in the coming decades.

\section{Role of Cartilage in OA Pathology}

$\mathrm{OA}$ is a chronic and debilitating disease of the articular joints, characterized by a complex disease process, whereby

*Address correspondence to this author at the Caring Medical and Rehabilitation Services, S.C. 715 Lake St., Ste. 600, Oak Park, IL 60301, USA; Tel: (708) 848-7789; Fax: (708) 848-7763;

E-mail:drhauser@caringmedical.com joints undergo recurring cycles of damage through a continuum of events that alter both joint structure and joint morphology. The catalyst for these events is the progressive loss of articular cartilage in the joint, beginning at the joint surface and progressing until there is full thickness loss. Once at this stage, other joint structures become affected in a domino-like effect, as joint space narrowing, subchondral bone remodeling, varying degrees of synovial inflammation, and osteophyte (bone spur or bony overgrowth) formation begins to occur, progressing until joint movement becomes noticeably restricted. The appearance of osteophytes has become the criterion for advanced OA since they form at the margins of joint surfaces and can usually be detected radiographically [6] hence, the diagnostic term radiographic $O A$.

When cartilage is healthy, it has a smooth lubricated surface, allowing the joint to slide over bone. Without this surface, the joint loses its gliding motion and becomes limited in its movement. This loss also causes the spaces between the joint and bone to narrow, exposing the underlying subchondral bone and precipitating a process of bone remodeling, in which the subchondral bone thickens and a poorly mineralized matrix develops. In cases of extensive bone remodeling, bone marrow lesions become apparent as the ends of bones begin to rub together $[7,8]$. When the rate of tissue breakdown in a joint begins to surpass that of tissue repair, 
the event disrupts the dynamic equilibrium between the two, leading to progressive joint failure and development of OA in the joint. The loss of cartilage in $\mathrm{OA}$ is characterized by a similar imbalance, whereby excessive matrix degradation begins to overwhelm matrix synthesis, precipitating full thickness loss of the tissue and the development of OA [911]. This theory reflects the importance of articular cartilage in joint homeostasis and the tissue's pivotal role in the pathogenesis of $\mathrm{OA}$ and its progression.

Articular cartilage is unique because it contains only one cell type the chondrocyte that is responsible for both the synthesis and breakdown of the tissue's cartilaginous extracellular matrix. Thus, when full thickness loss occurs, there is no cellular base or viable extracellular matrix from which new tissue can grow; this is especially true in the tissue's superficial zone at the joint surface where changes in cartilage first occur [12]. Nonetheless, there has been evidence that under some circumstances of chronic injury, such as that seen in OA, chondrocytes are capable of demonstrating a significant reparative response and can replicate their DNA to form new cells [13]. Analysis of cartilage from joints with OA has shown an increased number of cells in clones and evidence for DNA synthesis by such means as 3H-thymidine metabolic studies, autoradiography, and histological demonstration of mitotic figures [14-16].

Articular cartilage is also unique in that it is both avascular and aneural. Without a blood supply, cartilage tends to heal slowly and imperfectly; as long as the chondral lesion remains contained within the tissue, there is no involvement of the vasculature [17]. Since cartilage lacks a neural network as well, it does not elicit pain itself; instead, the pain in OA occurs when there is excessive pressure on the subchondral bone subsequent to the loss of cartilage tissue or when there are excessive stressors on periarticular structures such as ligaments and tendons [8].

Despite the myriad of medications and procedural techniques that have been developed over the years, there is still no cure for $\mathrm{OA}$ or a viable disease-modifying agent available $[6,9,11]$. Exercise, medications, physical therapy, and lifestyle modification may decrease symptoms and improve mobility, but they do not reverse the disease. The success rate for regenerating cartilage tissue for the most part has been relatively lackluster. Thus, OA continues to be treated symptomatically to relieve pain and improve function.

To that end, we took a pragmatic approach and canvassed a group of patients from our pain clinic who agreed to undergo bone marrow prolotherapy (BMP), a treatment that uses bone marrow aspirate (BMA) injection therapy in combination with hypertonic dextrose prolotherapy, and then to provide feedback via a self-reported questionnaire as to whether this combined treatment protocol was more effective than prolotherapy alone in reducing pain and improving function in osteoarthritic joints. The cohort we recruited was comprised of 24 patients who had been clinically diagnosed previously with radiographic OA by their attending physician. The results described herein were obtained in accordance with the guidelines of the Declaration of Helsinki.

\section{METHODS}

\section{Patients}

Our sample cohort was comprised of 24 adult patients $(12 \mathrm{M}, 12 \mathrm{~F})$ who had a diagnosis of radiographic OA and had visited our chronic pain clinic in 2009 for prolotherapy treatment to relieve their chronic pain. Patients were asked if they would be willing to receive bone marrow prolotherapy at prescribed intervals of 6 to 8 weeks and then were given an explanation of the combined treatment technique; they were also told they would be required to participate in an exercise program. In addition, at 6 months after completing the BMP treatment protocol, they would be given a questionnaire and asked to assess a number of variables using a numerical scoring system to rate their condition before and after treatment. All patients had been previously diagnosed with radiographic OA, either of the knee (13 patients), hip (8 patients), ankle (1 patient), or toe (1 patient), with the exception of one patient who had lower back pain from OA of the facet joints and degenerative disc disease. Average age of patients was 64.9, with an age range of 57 to 76 . Demographic data of the study participants are presented in Table 1.

Inclusion criteria for the study were the following: (1) established diagnosis of OA by a physician and x-ray or MRI evidence of advanced OA of the involved joint(s); (2) duration of pain greater than 6 months; (3) no other treatments specific to OA [eg, use of NSAIDs or corticosteroid injections, physical therapy]; (4) age of at least 18 years; (5) completion of the BMP treatment protocol; (6) compliance with a prescribed exercise program; (7) available for followup 6 months post-treatment; and (8) completion of a patient questionnaire regarding the results of BMP treatment. $\mathrm{Pa}-$ tients who were diagnosed with other conditions affecting the joints or with systemic diseases, who were undergoing concomitant therapy, or who were taking over-the-counter or prescription medications, NSAIDs, steroid preparations, or narcotics were excluded from the study.

All patients selected underwent BMP treatment sessions (average 3.6) conducted every 6 to 8 weeks and were interviewed about their treatment following the final BMP session. Six months after completion of BMP treatment, patients were sent a questionnaire by e-mail asking them to assess their condition before and six months after treatment.

\section{Treatment Protocol and Procedures}

Before treatment began, all patients reviewed and signed a consent form, which described the inherent benefits and risks associated with the combined BMP procedure and were asked if they understood the treatment regimen. Vitals for each patient were recorded and medical history was obtained and documented. All patients were to receive a prescribed series of BMP treatments comprised of injections with a hypertonic dextrose solution at a predetermined number of sites in and around the index joint per session every 6 to 8 weeks and at least two treatment sessions of BMA injections in and around said joint, unless the desired clinical response occurs earlier.

Prolotherapy itself is a technique that is used to aid the body in healing by prompting it to create new collagen. A solution, usually of hypertonic dextrose, is injected into an 
injury site to elicit localized inflammation, which is the first step in healing the damaged area. The dextrose solution acts as a proliferant since the inflammation it causes stimulates the fibroblast cells that make collagen, reticular and elastic fibers, glycosaminoglycans, and glycoproteins, all of which are found in the extracellular matrix. With the addition of BMA injections, the goal is to enhance the effects of hypertonic dextrose prolotherapy and improve treatment outcomes in patients with advanced osteoarthritic conditions.

Combined BMP treatment was carried out as shown in Fig. (1), according to the procedure described in the steps that follow.
The area in and around the joint(s) to be treated was cleaned with $4 \%$ chlorhexadine gluconate and then anesthetized with 5\% lidocaine cream. After 10-15 minutes, the area was cleaned with $3 \%$ hydrogen peroxide and then with the $2 \%$ chlorhexadine gluconate solution. For all prolotherapy treatments, the injectant contained $15 \%$ dextrose, $0.1 \%$ procaine, and $10 \%$ sarapin.

For knee prolotherapy, patients remained in the supine position and $1 \mathrm{cc}$ of $8 \%$ procaine was injected intraarticularly into the index knee, followed by an injection of 4 cc $1 \%$ procaine solution into the primary sites of pain where the bone marrow was to be injected. The anterior aspect of

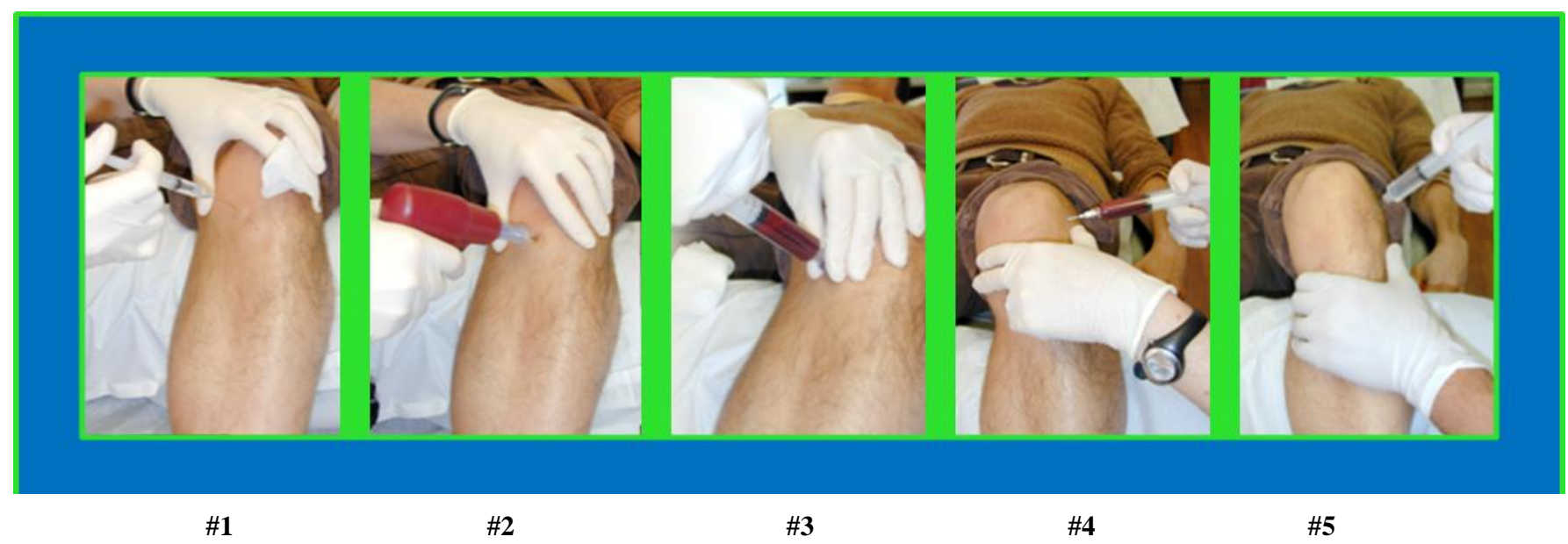

Fig. (1). Procedure for direct bone marrow prolotherapy

\#1 Procaine, or other suitable numbing agent, is injected into the tibia to anesthetize the site of the bone marrow extraction.

\#2 An EZIO drill, is used to pierce the tibial bone for extraction of the bone marrow.

\#3 The bone marrow is drawn from the tibial bone.

\#4 The entire bone marrow aspirate is then injected directly into the knee joint or other injury site.

\# 5 Following the bone marrow treatment, standard prolotherapy is performed around the entire joint to accelerate the growth and repair of the surrounding ligaments and tendons.

*Steps 4 and 5 are intechangeable.

For bone marrow harvesting, patients were placed in the supine position and the medial tibial area to be aspirated was cleaned with a solution of $2 \%$ chlorhexadine gluconate and $70 \%$ isopropyl alcohol and then anesthetized with $4 \mathrm{cc}$ of $1 \%$ procaine solution, which was administered intradermally, subcutaneously, and on the periosteum around the aspiration site, approximately $5 \mathrm{~cm}$ inferior to the tibial plateau and 3 $\mathrm{cm}$ lateral to the midline. The area was cleaned again with the chlorhexadine solution. Bone marrow access was obtained using an EZ-IO intraosseous drill with a $45 \mathrm{~mm}$ needle. Once the periosteum was pierced, the stylet was removed and a $12 \mathrm{cc}$ lock syringe containing 3,000 IU of heparin was used to aspirate $8 \mathrm{cc}(\mathrm{X} 2)$ of bone marrow, after which the syringe was detached; an empty $10 \mathrm{cc}$ syringe was attached, and the needle was removed via negative pressure. The area was covered with a pressure dressing. Generally, only one bone marrow injection is administered directly into any given joint; a second aliquot of BMA was injected into tissue around the joint.

All patients were prepped for BMP injection therapy; knee patients were placed in the supine position and hip patients, in the prone position and then in the supine position. the joint (right or left knee) was injected with a total of $40 \mathrm{cc}$ of the dextrose solution at 24 locations, specifically at the site of bony attachments in and around the medial and lateral collateral ligaments, patellar ligament, vastus medialis, iliotibial tract, and pes anserinus. Following this procedure, 8 cc of the BMA previously extracted was injected intraarticularly into the knee joint. A second aliquot of BMA was injected into tissue around the joint at areas of pain.

For hip prolotherapy, the anterior, lateral and posterior regions of the hip were treated. Patients were placed in the prone position and the area to be treated was cleaned with $3 \%$ hydrogen peroxide, followed by the $2 \%$ chlorhexadine gluconate solution. Then, the dextrose prolotherapy solution was injected into the following areas in the posterior hip, including the greater trochanter, intertrochanteric crest, neck of femur, and dorsal ischium: ischiofemoral and iliofemoral ligaments; tensor fascia lata; gluteus medius, pyriformis, gemellus superior, quadratus femoris, obturator internus, gemellus inferior, and vastus lateralis muscles. Then, using the lateral approach, $8 \mathrm{cc}$ of BMA was injected intraarticularly and another $8 \mathrm{cc}$ peri-articularly at areas of pain. 
Table 1. Demographic Data of Study Cohort: Patient Characteristics and Medical History

\begin{tabular}{|c|c|c|c|}
\hline Patient No. $(\mathrm{N}=24)$ & Age (Mean: 64.9) & Diagnosis & Prior Recommendation for Surgery \\
\hline 1 & 61 & R knee OA & Partial removal, meniscus \\
\hline 2 & 59 & $\mathrm{R}$ knee, torn medial meniscus post surgery & Removal, medial meniscus \\
\hline 3 & 60 & Avascular necrosis of talus, OA of ankle & Pin insertion in ankle, donor cartilage \\
\hline 4 & 69 & R knee OA & Knee replacement \\
\hline 5 & 69 & L knee OA & Knee replacement \\
\hline 6 & 76 & R hip OA & Hip replacement \\
\hline 7 & 76 & L hip OA & Hip replacement \\
\hline 8 & 61 & L knee OA & NA \\
\hline 9 & 74 & L hip OA & Hip replacement \\
\hline 10 & 63 & R knee OA & Knee replacement \\
\hline 11 & 63 & L knee OA & Knee replacement \\
\hline 12 & 71 & R knee OA & Knee replacement \\
\hline 13 & 71 & L knee OA & Knee replacement \\
\hline 14 & 57 & R knee OA & NA \\
\hline 15 & 57 & L knee OA & NA \\
\hline 16 & 57 & R hip OA & Hip replacement or steroids \\
\hline 17 & 57 & L hip OA & NA \\
\hline 18 & 70 & L hip OA & Hip replacement \\
\hline 19 & 70 & R hip OA & Hip replacement \\
\hline 20 & 70 & $\mathrm{~L}$ big toe OA with bone spur & Bone fusion \\
\hline 21 & 63 & R hip OA & NA \\
\hline 22 & 63 & Lower back pain from degenerative disc disease & NA \\
\hline 23 & 60 & R knee OA & Knee replacement \\
\hline 24 & 60 & L knee OA & Knee replacement \\
\hline
\end{tabular}

Patients were then turned over and placed in the supine position. The area to be treated was cleaned with $3 \%$ hydrogen peroxide, followed by the $2 \%$ chlorhexadine gluconate solution. The following structures were injected with hypertonic dextrose prolotherapy solution at their bony attachments around the anterior portion of the right greater trochanter and hip joint: iliofemoral and pubofemoral ligaments, hip labrum, and gluteus minimus muscle. For the two-sided hip procedure, a total of $60 \mathrm{cc}$ of solution was used in 42 separate injections.

Three patients had BMP injections in other areas. Patients No. 3 and No. 20 had osteoarthritis of the ankle subsequent to ankle avascular necrosis of the talus and severe osteoarthritis of the left big toe, respectively. Patient No. 3 had $3 \mathrm{cc}$ of BMA injected into the subtalar and tibiotalar joints and another $8 \mathrm{cc}$ of BMA injected into the medial and lateral ligament ankle complexes. Patient No. 20 had $1 \mathrm{cc}$ of BMA injected into the metatarsophalangeal joint and an additional $3 \mathrm{cc}$ of BMA injected around the joint. Because the areas to be treated in these two patients did not require as much solution as patients with knee and hip OA, the primary injectant was BMA, although patient No. 3 did receive some dextrose prolotherapy to areas not treated with BMA-namely, the posterior talofibular ligaments and the small ligaments around the ankle (eg, talonavicular and plantar calcaneonavicular). Patient No. 22 had unresolved low back pain from L4-S1 degenerative arthritis (degenerative disc disease and facet arthritis or degeneration in those areas bilaterally) and had $8 \mathrm{cc}$ of BMA injected into and around the L4-S1 facet joints and another $4 \mathrm{cc}$ of BMA into each sacroiliac joint. In this patient, dextrose prolotherapy (total: $40 \mathrm{cc}$ of 
solution/24 injections) was also utilized to treat the sacroiliac and iliolumbar ligaments.

Patients tolerated the procedures well and were left to rest for 10-15 minutes with moist heat covering the treated joint(s), after which the area was cleaned with $3 \%$ hydrogen peroxide.

At follow-up, at least 6 months after completing BMP treatment, patients were e-mailed a self-assessment questionnaire consisting of 27 questions, including those related to the severity of their conditions at baseline and after BMP treatment, to their level of pain (Visual Analog Pain Scale) while at rest, performing activities of daily living (ADL), and during exercise, their ability to perform ADL, their daily range of motion (ROM), their degree of stiffness, and their level of crepitus (crunching). Patients were asked to rate these variables using a scale of 0 to 3 for minimal, 4 to 6 for moderate, and 7 to 10 for severe before and 6 or more months after BMP treatment. Patients were also asked to assess their daily pain level after treatment in terms of lasting improvement, given as a percentage. Primary outcome measures were improvements in pain levels and joint function.

\section{RESULTS}

Before coming to our pain clinic for treatment, 18 of the 24 participants $(75 \%)$ enrolled in the study had already been advised to have surgery by another physician, 14 of whom had been told they needed knee or hip replacement (see Table 1). All data presented herein and in Table 2 are based on responses elicited 6 or more months after each patient had completed the prescribed series of injection treatments. Outcome measures were the changes perceived by patients before and after BMP treatment (average number of sessions, 3.6) regarding the following: pain levels while at rest, while performing ADL, and while doing exercise; degree of stiffness; limitation in ROM; and level of crepitus. Data from outcomes were obtained using GraphPad statistical software (Prism) and analyzed against baseline utilizing a paired student t test, with $P=.05$ considered as significant. Two patients who had resolution of the majority of their symptoms with only one visit were included in the data analysis; a twenty-fifth patient was lost to follow-up and was not included in the data analysis.

\section{Pain Level}

Participants in the study were asked to rate their pain level before and after BMP treatment under three conditions: at rest, performing $\mathrm{ADL}$, and during exercise, using a scale of 0 to 10 based on the Visual Analog Pain Scale (VAS), in which 0 to 3 was minimal pain, 4 to 6 , moderate pain, and 7 to 10 , severe pain. Of the 13 patients who had knee OA, 10 patients $(77 \%)$ reported having no pain at rest after BMP treatment; 9 patients $(69 \%)$ reported no pain during ADL; and 7 patients $(54 \%)$ reported no pain during exercise. Of the 8 patients who had hip OA, all $8(100 \%)$ reported having no pain at rest after treatment; 7 patients $(88 \%)$ had no pain during ADL; and 6 patients $(75 \%)$ reported no pain on exercising. Of the 3 patients diagnosed with other types of joint disease, all 3 patients (100\%) reported having no pain at rest after BMP treatment; again all 3 patients $(100 \%)$ reported having no pain while performing ADL; and all 3 patients $(100 \%)$ reported having no pain on exercising. The amelioration of pain at rest, performing ADL, and during exercise after BMP treatment was statistically significant $(P<.001)$, as shown by two-tailed tests of paired samples comparing the differences between baseline and post-treatment scores in pain levels (Table 2).

\section{Degree of Stiffness}

Patients were asked to describe their degree of stiffness before and after BMP treatment on a scale of 0 to 10 , with 0 being no stiffness and 10 being extremely stiff. Of the 13 knee OA patients, 7 patients $(54 \%)$ reported having no stiffness after BMP treatment; of the 8 hip OA patients, 4 had no stiffness and another 4, minimal stiffness (100\%) after BMP treatment; and of the 3 patients diagnosed with other types of joint disease, 2 patients $(67 \%)$ had minimal stiffness. The amelioration of stiffness after BMP treatment was statistically significant $(P<.001)$ as shown by two-tailed tests of paired samples comparing the differences between baseline and post-treatment scores in rating degree of stiffness (Table 2).

\section{Range of Motion}

Patients were asked to rate their average daily ROM level before and after BMP treatment on a scale of 0 to 10 , with 0 being no lack of ROM and 10 being severe limitation in ROM. Of the 13 knee OA patients, 9 patients (69\%) reported having no ROM problem after BMP treatment; of the 8 hip OA patients, 2 patients reported having no ROM problem and another 2, only minimal loss in ROM (50\%) after BMP treatment; and of the 3 patients diagnosed with other types of joint disease, 2 patients $(67 \%)$ reported minimal loss in ROM. The improvement in ROM after BMP treatment was statistically significant $(P<.001)$ as shown by two-tailed tests of paired samples comparing the differences between baseline and post-treatment scores in rating daily ROM (Table 2).

\section{Level of Crepitus}

Patients were asked to rate their average daily level of crunching (crepitus) before and after BMP treatment on a scale of 0 to 10 , with 0 being no crunching and 10 being severe crunching. Of the 13 knee OA patients, 10 patients reported no crunching and 3, minimal crunching (100\%) after BMP treatment. Of the 8 hip OA patients, 7 patients had no crunching and the remaining patient, only minimal crunching (100\%) after BMP treatment,. Of the 3 patients diagnosed with other types of joint disease, all 3 patients (100\%) reported having no crunching after BMP treatment. The decreased level of crepitus after BMP treatment was statistically significant $(P<.001)$ as shown by two-tailed tests of paired samples comparing the differences between baseline and post-treatment scores in rating levels of crunching (Table 2). 
Table 2. Self-assessment of Pain and Function in Patients with OA Before and Six months after Bone Marrow Prolotherapy

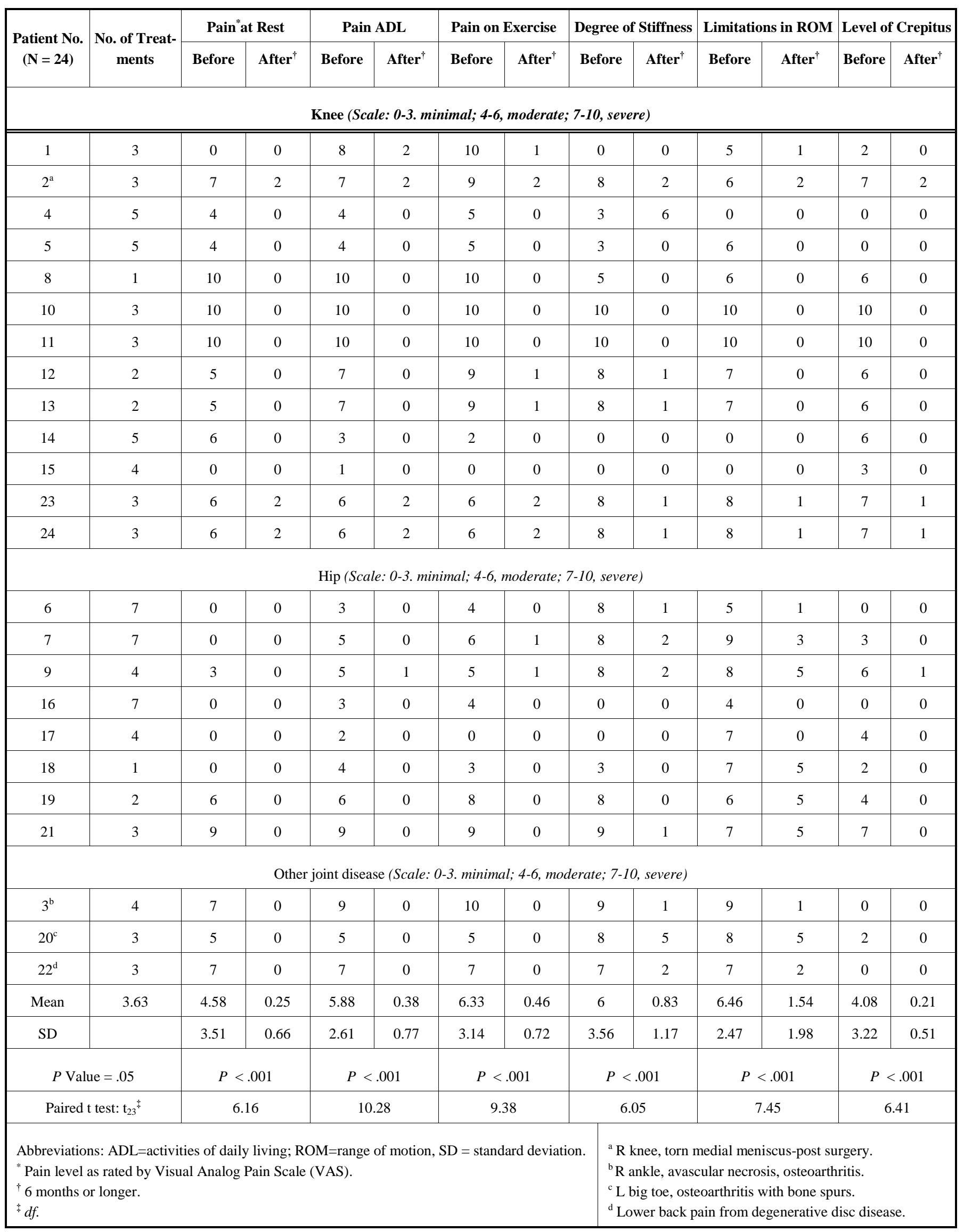




\section{Overall Improvement}

Patients were also asked to rank their daily ability to exercise symptom-free before and 6 months after BMP treatment, with "a" being no exercising restrictions and " $\mathrm{e}$ " being totally compromised (letters were transformed to numbers 1 to 5 for analysis). Average score at baseline before treatment was 3.63 (ie, at the high end of being moderately compromised when exercising). After BMP treatment, the average score was 1.54 (ie, midway on the scale to having no restrictions in exercising). This represents a 2.4 times increase inthe ability of patients to exercise symptom-free 6 or more months after BMP treatment. Patients were then asked to rank their daily sustained improvement overall as a percentage in regard to pain levels, stiffness, ROA, and crepitus at six months or more after completion of all BMP treatments. Of the 13 knee OA patients, 10 reported $100 \%$ improvement after completing therapy, and the remaining 3 , rated it as $97 \%, 90 \%$, and $90 \%$, respectively. Of the 8 hip OA patients, 7 reported $100 \%$ improvement and the remaining patient, only $75 \%$. All 3 patients diagnosed with other types of joint disease reported $100 \%$ improvement in their condition after treatment.

\section{DISCUSSION}

BMP treatment or prolotherapy alone comprises only one facet of a comprehensive prescription protocol we follow at our chronic pain clinic in order to promote the acceleration of the body's natural response to recovery and achieve resolution of pain and other debilitating symptoms associated with OA or cartilage-related conditions. Aside from receiving all of the full number of BMP or prolotherapy treatments, we educate and advise patients beforehand on lifestyle issues to follow-namely, getting the recommended amount of sleep, eating a healthy diet, taking supplements wisely, refraining from taking NSAID pain medication, adhering to the prescribed ROM and strengthening exercises (eg, cycling), and avoiding activities that could cause reinjury.

In the questionnaire patients completed, they were also queried on how closely they adhered to this comprehensive regimen while undergoing BMP treatment. Of the 24 participants in the study, 23 said they were $75 \%$ or more compliant in following the comprehensive prescribed regime, 14 (58\%) of which said they were $95 \%$ to $100 \%$ disciplined; only one respondent indicated adherence was "not so good". As to overall satisfaction, all participants were asked if BMP met their expectations. Of the 24 participants in the study, 23 said they were satisfied with the outcome of their treatment; there was one non-responder.

Patient-reported outcomes from the current study are in agreement with those of a similar study we conducted in our clinic using BMP injection therapy [18]. In that case series, nearly all 7 patients experienced significant gains up to 12 months after treatment without experiencing adverse events; in 5 of the cases, patients reported having gained either considerably more functional improvement or total to almost total pain relief. In 3 of the cases, patients who had been treated previously with prolotherapy alone but had reached a plateau achieved substantial gains again after BMP combina- tion treatment. In follow-up interviews, patients were unanimous in expressing satisfaction with treatment outcomes.

\section{The Potential of Prolotherapy}

Prolotherapy is an injections therapy for chronic musculoskeletal pain and is an age-old technique adapted for use by mainstream medicine by George S. Hackett, MD in the 1940s. Although the technique has largely been thought of as alternative medicine, a number of studies, including recently published randomized controlled trials (RTC) [19-24], are bringing prolotherapy to the forefront and demonstrating that it does indeed offer benefits in terms of pain relief, regenerative properties, and cartilage repair for people affected by musculoskeletal disorders such as OA.

Dumais, et al. [25] report that the use of a dextrose/lidocaine solution as regenerative injection therapy (RIT) to relieve pain and restore function in patients with knee osteoarthritis is associated with a marked reduction in symptoms which was sustained for over 24 weeks, as shown in a crossover study in which patients were randomly assigned to receive RIT in combination with exercise therapy, either during the beginning weeks of treatment or during the latter weeks. The improvement attributable to RIT alone corresponds to an 11.9-point (or 29.5\%) decrease in the Western Ontario and McMaster Universities Osteoarthritis Index (WOMAC) scores.

In a 3-arm, blinded, randomized controlled trial, Rabago and colleagues [26] report that patients with knee OA who were treated with dextrose prolotherapy also had clinically meaningful and sustained improvements in pain, function, and stiffness scores. WOMAC scores for the intervention group improved more $(P<.05)$ at 52 weeks than those for the saline or exercise groups and exceeded the WOMACbased minimal clinically important difference.

In two earlier placebo-controlled double-blind studies, prolotherapy was shown to have beneficial effects on OA, including evidence of X-ray changes [27,28]. These results concur with experience in our own pain clinic, in that we were able to show that cartilage regeneration occurred after prolotherapy treatment in five knees showing articular cartilage degeneration. In all five cases, X-ray findings after treatment showed detectable increases in joint space width (JSW) in each of the treated knees, signifying the regeneration of articular cartilage. These increases coincided with improvements in pain level and function [29]. (See Fig. 2).

\section{Mesenchymal Stem Cells vs. Direct Bone Marrow}

Over the last two decades, the use of adult mesenchymal stem cells (MSCs) has generated intense interest within the medical community due to the cell's ability to differentiate into multiple cell lineages, particularly those of cartilage, muscle, and bone. To obtain a sufficient pool of MSCs from autologous bone marrow aspirates, centrifugal concentration or expansion through in vitro culturing has been the norm. However, numerous studies [7, 30-35] have since shown that manipulating MSCs to derive a better yield has its drawbacks and can affect the quality of the pool. 

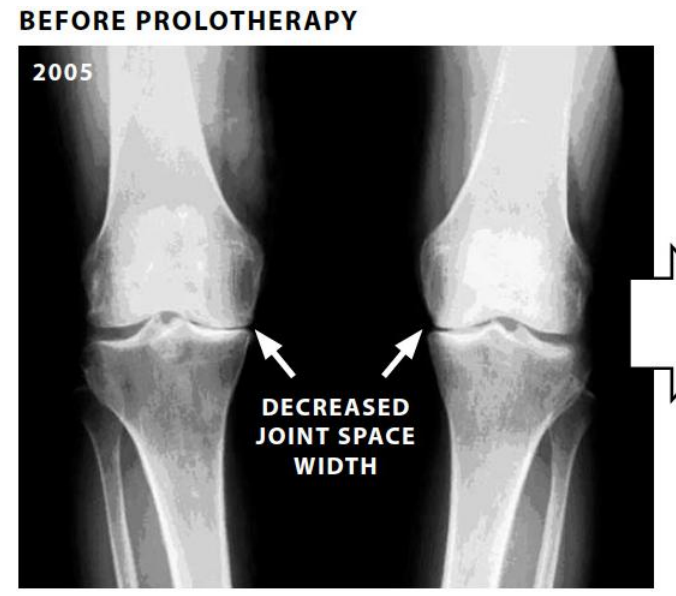

\section{AFTER PROLOTHERAPY}

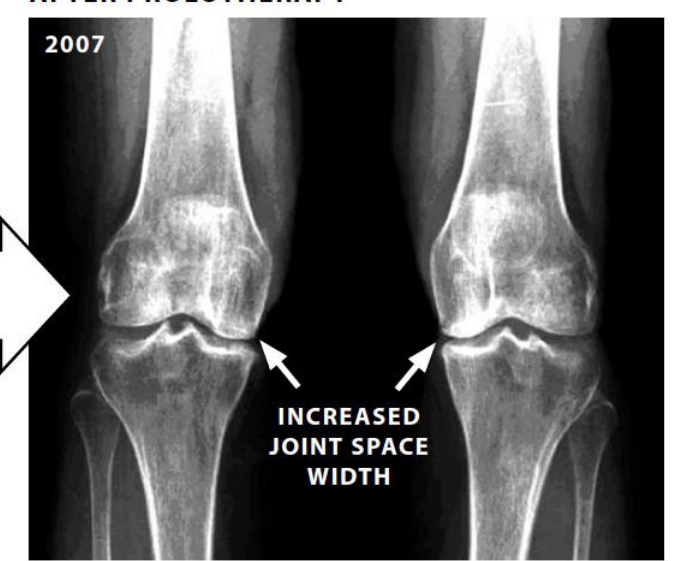

Fig. (2). Standard weight bearing bilateral knee $X$-rays before and after prolotherapy.

The widening of the medial joint space width in both knees (shown by arrows) indicates that cartilage regeneration has taken place.

An alternate approach, which has shown promise in preliminary studies [36-42], is to treat diseased joints with autologous chondrogenic bone marrow-derived MSCs (BMSCs), prepared as a buffy-coat fraction of bone marrow. With the use of direct tibial WBM, it is possible to capture elements of regenerative strategies that other therapies do not. Because the marrow is not fractionated in WBM, vital chondrogenic components in the marrow plasma can be retained in addition to the pool of BMSCs. In essence, WBM injections mimic the bone marrow's natural niche microenvironment, in that all key cells remain in their natural ratios, allowing for better cellular viability and higher proliferative and regenerative potentials. Duygulu and colleagues [43] have demonstrated histologically the regenerative effects achieved with intra-articular administration of autologous bone marrow aspirate.

Therefore, we theorized that using direct injection of unfractionated native whole BMA with dextrose prolotherapy would enhance treatment effects. We then surveyed patients who had agreed to undergo BMP treatment as described herein to determine if such a strategy would be more effective in alleviating their symptoms. Preliminary results and observations of using BMP injection therapy for treatment of osteoarthritic joints suggest that the procedure is potentially efficacious as well as safe, with no known deleterious effects reported to date. Limitations of the study include its small size and subjective nature, as well as its lack of a control or comparative group, and the absence of radiographic evidence.

\section{CONCLUSION}

The results of our study have shown that a combined BMP treatment regimen of injections to index sites has an enhanced effect on outcomes, as perceived by patients themselves, in regard to pain relief and improved joint function and has the potential to improve the QoL of individuals with radiographic OA. Patients treated with BMP reported significantly decreased pain and significantly increased function $(P<.001)$ in all areas measured, including gains in ADL, exercise ability, and ROM, as well as losses in stiffness and crepitus. Furthermore, we propose that BMP treatment has the potential to slow and possibly reverse progression of OA in joints by promoting the regeneration of articular cartilage. Should that be the case, a new paradigm in the treatment of OA may be possible, but additional research and more highly powered studies which include evidence of radiological changes are required.

\section{DECLARATION OF CONFLICTING INTERESTS}

The author(s) declared the following potential conflicts of interest with respect to the research, authorship, and/or publication of this article: RH declares that he is co-founder of Caring Medical \& Rehabilitation Services, S.C. which provides prolotherapy services. BW does not have any conflict of interest to declare.

\section{LOCATION WHERE STUDY WAS CONDUCTED}

Caring Medical and Rehabilitation Services, S.C. Oak Park, IL

\section{FUNDING}

The authors received no financial support in conducting the research or carrying out the study reported in this article.

\section{REFERENCES}

[1] Arthritis Foundation. OA research initiative: seeking a cure. Available at http://www.arthritis.org/research/research-focus/researchoa-initiative/ [Accessed 2013 January 4].

[2] Symmons D, Mathers C, Pfleger B. Global burden of disease 2000. World Health Organization World Health Report. Geneva: World Health Organization 2002.

[3] World Health Organization. Chronic diseases and health promotion: chronic rheumatic conditions. Available at http://www.who.int/chp/topics/rheumatic/en/ [Accessed May 8, 2013].

[4] Gruber WH, Hunter DJ. Transforming osteoarthritis care in an era of health care reform. Clin Geriatr Med 2010; 26: 433-44.

[5] National Institute of Arthritis and Musculoskeletal and Skin Diseases, National Institutes of Health. Osteoarthritis initiative. Available at http://www.niams.nih.gov/funding/funded_research/ osteoarthritis_initiative/default.asp [Accessed May 22, 2013].

[6] Matthews GL. Disease modification: promising targets and impediments to success. Rheum Dis Clin N Am 2013; 39: 177-87.

[7] Lubis AMT, Lubis VK. Adult bone marrow stem cells in cartilage therapy. Acta Medica Indonesiana - Indonesian J Int Med 2012; 44 (1): 62-8. 
[8] Loeser RF. Age-related changes in the musculoskeletal system and the development of osteoarthritis. Clin Geriatr Med 2010; 26: 37186.

[9] Pulsatelli L, Addimanda O, Brusi V, Pavloska B, Meliconi R. New findings in osteoarthritis pathogenesis: therapeutic implications. Ther Adv Chronic Dis 2013; 4: 23-43.

[10] Meszaros E, Malemud CJ. Prospects for treating osteoarthritis. Ther Adv Chronic Dis 2012; 3(5): 219-29.

[11] Hunter DJ, Graverand-Gastineau HL. How close are we to having structure-modifying drugs available? Rheum Dis Clin N Am 2008; 34: 789-802.

[12] Hollander AP, Dickinson SC, Kafienah W. Stem cells and cartilage development: complexities of a simple tissue. Stem Cells 2010; 28 : 1992-6.

[13] Redman S, Dowthwaite GP, Thomson BM, Archer CW. The cellular responses of articular cartilage to sharp and blunt trauma. Osteoarthr Cartil 2004; 12: 106-16.

[14] Telhag H. DNA synthesis in degenerated and normal joint cartilage in full-grown rabbits. Acta Orthop Scand 1973; 44: 604-10.

[15] Rothwell A, Bentley G. Chondrocyte multiplication in osteoarthritis Articular Cartilage. J Bone Joint Surg 1973; 55B: 588-94.

[16] O'Driscoll S. Current concepts review- The healing and regeneration of articular cartilage. J Bone Joint Surg 1998; 80: 1795-812.

[17] Longo UG, Petrillo S, Franceschetti E, Berton A, Maffulli N, Denaro V. Stem cells and gene therapy for cartilage repair. Stem Cells Int 2012; 2012; 168385 .

[18] Saraf A, Mikos AG. Gene delivery strategies for cartilage tissue engineering. Adv Drug Deliv Rev 2006; 58 (4): 592-603.

[19] Trippel SB. Growth factor actions on articular cartilage. J Rheum 1995; 22 (1): 129-32.

[20] Hauser R, Amos Orlofsky. Regenerative injection therapy with whole bone marrow aspirate for degenerative joint disease: a case series. Clin Med Insights: Arthritis Musculoskeletal Disord. (In press).

[21] Rabago D, Zgierska A, Fortney L, et al. Hypertonic dextrose injections (prolotherapy) for knee osteoarthritis: results of a single-arm uncontrolled study with 1-year follow-up. J Altern Complement Med 2012; (4): 408-14.

[22] Uthman I, J-P Raynauld J-P, Haraoui B. Intra-articular therapy in osteoarthritis. Postgrad Med J 2003; 79: 449-53.

[23] Best TM, Zgierska AE, Zeisig E, Ryan M, Crane D. A systematic review of four injection therapies for lateral epicondylosis: prolotherapy, polidocanol, whole blood and platelet rich plasma. $\mathrm{Br}$ J Sports Med 2009; 43 (7): 471-81.

[24] Rabago D, Slattengren A, Zgierska A. Prolotherapy in primary care practice. Prim Care 2010; 37 (1): 65-80.

[25] Dumais R, Benoit C, Dumais A, et al. Effect of regenerative injection therapy on function and pain in patients with knee osteoarthritis: a randomized crossover study. Pain Med 2012; (8): 990-9.

[26] Rabago D, Patterson JJ, Mundt M, et al. Dextrose prolotherapy for knee osteoarthritis: a randomized controlled trial. Ann Fam Med 2013; 11 (3): 229-37.

[27] Barkan A. Acromegalic arthropathy and sleep apnea. J Endrocrinol 1997; 155: S41-S44.

[28] Chrisman OD. The effect of growth hormone on established cartilage lesions. Clin Orthoped 1975; 107: 232-8

[29] Standard Clinical X-ray Studies Document Cartilage Regeneration in Five Degenerated Knees After Prolotherapy. Hauser RA, Cukla
JJ. Available at http://www.journalofprolotherapy.com/index.php/ standardclinical-x-ray-studies-document-cartilage-regeneration-infive-degenerated-knees-after-prolotherapy/\#sthash.iEBqlT8D.dpuf [Accessed Oct. 22, 2013].

[30] Binato R, de Souza Fernandez T, Lazzarotto-Silva C, et al. Stability of human mesenchymal stem cells during in vitro culture: considerations for cell therapy. Cell Prolif 2013; 46: 10-22.

[31] Fossett E, Khan WS. Optimising Human Mesenchymal Stem Cell Numbers for Clinical Application: A Literature Review. Stem Cells Int 2012; 2012: 456259 .

[32] Rodrigues M, Blair H, Stockdale L, Griffith L, Wells A. Surface tethered epidermal growth factor protects proliferating and differentiating multipotential stromal cells from FasL-induced apoptosis. Stem Cells 2013; 31: 104-16.

[33] Sekiya I, Larson BL, Smith JR, Pochampally R, Cui J-G, Prockop DJ. Expansion of human adult stem cells from bone marrow stroma: conditions that maximize the yields of early progenitors and evaluate their quality. Stem Cells 2002; 6: 530-41.

[34] Solchaga LA, Penick KJ, Welter JF. Chondrogenic differentiation of bone marrow-derived mesenchymal stem cells: tips and tricks. Methods Mol Biol 2011; 698: 253-78.

[35] Dominici M, Le Blanc K, Mueller I, et al. Minimal criteria for defining multipotent mesenchymal stromal cells: the International Society for Cellular Therapy position statement. Cytotherapy 2006; 8 (4): 315-7.

[36] Gobbi A, Karnatzikos G, Scotti C, Mahajan V, Mazzucco L, Grigolo B. One-step cartilage repair with bone marrow aspirate concentrated cells and collagen matrix in full-thickness knee cartilage lesions: results at 2-year follow-up. Cartilage 2011; 2: 286-99.

[37] Nejadnik H, Hui JH, Choong EPF, Tai BC, Lee EH. Autologous bone marrow-derived mesenchymal stem cells versus autologous chondrocyte implantation: an observational cohort study. Am J Sports Med 2010; 38: 1110-6.

[38] Ossendorf C, Steinwachs MR, Kreuz PC, et al. Autologous chondrocyte implantation (ACI) for the treatment of large and complex cartilage lesions of the knee. Sports Med Arthrosc Rehabil Ther Technol 2011; 3: 11-5.

[39] Davatchi F, Abdollahi BS, Mohyeddin M, Shahram F, Nikbin B. Mesenchymal stem cell therapy for knee osteoarthritis: preliminary report of four patients. Int J Rheum Dis 2011; 14: 211-5.

[40] Centeno CJ, Busse D, Kisiday J, Keohan C, Freeman M, Karli D. Increased knee cartilage volume in degenerative joint disease using percutaneously implanted, autologous mesenchymal stem cells. Pain Phys 2008; 11: 343-53.

[41] Emadedin M, Aghdami N, Taghiyar L, et al. Intra-articular injection of autologous mesenchymal stem cells in six patients with knee osteoarthritis. Arch Iran Med 2012; 15 (7): 422-8.

[42] Getgood A, Henson F, Skelton C, et al. The augmentation of a collagen/glycosaminoglycan biphasic osteochondral scaffold with platelet-rich plasma and concentrated bone marrow aspirate for osteochondral defect repair in sheep: a pilot study. Cartilage 2012; 3 (4): 351-63.

[43] Duygulu F, Demirel M, Atalan G, et al. Effects of intra-articular administration of autologous bone marrow aspirate on healing of full-thickness meniscal tear: an experimental study on sheep. Acta Orthop Traumatol Turc 2012; 46 (1): 61-7.

(C) Hauser and Woldin; Licensee Bentham Open.

This is an open access article licensed under the terms of the Creative Commons Attribution Non-Commercial License (http://creativecommons.org/licenses/ by-nc/3.0/) which permits unrestricted, non-commercial use, distribution and reproduction in any medium, provided the work is properly cited. 